\begin{tabular}{|c|c|}
\hline Title & Finite Element A nalysis of Magnetically Shielded Wire Coils U sing Homogenization Method \\
\hline Author(s) & Fujita, Shogo; Igarashi, Hajime \\
\hline Citation & $\begin{array}{l}\text { IEEE transactions on magnetics, 54(3), } 7401104 \\
\text { https://doi.org/10.1109/T MA G.2017.2758445 }\end{array}$ \\
\hline Issue Date & $2018-03$ \\
\hline Doc URL & http:/hdl.handle.net/2115/68965 \\
\hline Rights & $\begin{array}{l}\text { (C } 2017 \text { IEEE. Personal use of this material is permitted. Permission from IEEE must be obtained for all other uses, in } \\
\text { any current or future media, including reprinting/republishing this material for advertising or promotional purposes, } \\
\text { creating new collective works, for resale or redistribution to servers or lists, or reuse of any copyrighted component of } \\
\text { this work in other works. }\end{array}$ \\
\hline Tyре & article (author version) \\
\hline File Information & Finite Element A nalysis of Magnetically Shielded Wire Coils U sing Homogenization Method.pdf \\
\hline
\end{tabular}

Instructions for use 


\title{
Finite Element Analysis of Magnetically Shielded Wire Coils Using Homogenization Method
}

\author{
Shogo Fujita ${ }^{1}$, Hajime Igarashi ${ }^{1}$, Member, IEEE \\ ${ }^{1}$ Graduate School of Informatics Science and Technology, Hokkaido University, Sapporo, 060-0184
}

\begin{abstract}
A magnetically shielded wire (MSW), which has magnetic-conductive thin layers on the surface of a wire conductor, is expected to reduce the eddy current losses due to the proximity effect. The conventional finite element method (FEM) needs unacceptably long computational time to analyze eddy currents in multi-turn MSWs. This paper proposes a homogenization method which models MSW as a uniform material with complex permeability. The coil impedance evaluated by the homogenization-based FEM with coarse elements is shown to agree well with that obtained by the conventional FEM with much finer elements. Moreover, the optimal shield thickness is determined by the proposed method.
\end{abstract}

Index Terms-Magnetically shielded wire, proximity effects, homogenization method, complex permeability

\section{INTRODUCTION}

$\mathrm{D}$ evelopment of power devices composed of wideband semiconductors such as $\mathrm{SiC}$ and $\mathrm{GaN}$ allows us to increase switching frequency in electrical machines. This results in, however, increase in eddy current losses in their coils due to the skin and proximity effects. A magnetically shielded wire (MSW) is a wire conductor coated by magnetic-conductive thin layers [1]. When MSW is immersed in magnetic induction, magnetic fluxes tend to pass not through the central conductor but through the shielding layers. For this reason, the losses due to the proximity effect are expected to be reduced.

In order to compute the eddy current losses in MSWs by the conventional finite element method (FEM), they have to be discretized into fine elements which are sufficiently smaller than the skin depth. This leads to a large scale FE system of equations which needs large computational cost and storage.

Homogenization-based FEM has been shown to effectively analyze fine-structured materials [2-5]. When using method in $[4,5]$, MSW can be modeled as a uniform material with the homogenized complex permeability which represents eddy current loss and diamagnetic property caused by the proximity effect. This method has been shown to be effective for analysis of a multi-turn MSW coil under the assumption that the magnetic shield is insulator [5]. However, magneticconductive material is used for actual MSW [1]. A simple extension of the method in [5] for the analysis of actual MSW leads to numerical difficulty as will be shown later. In this paper, we propose a new method to analyze MSW with magnetic-conductive shielding layers without numerical difficulty.

\section{ANALYTICAL APPROACH}

In this section, the approach in $[4,5]$ is extended to analyze

Manuscript received June 27, 2017; Corresponding author: S.Fujita (email: fujita@em.ist.hokudai.ac.jp).

Digital Object Identifier (inserted by IEEE).

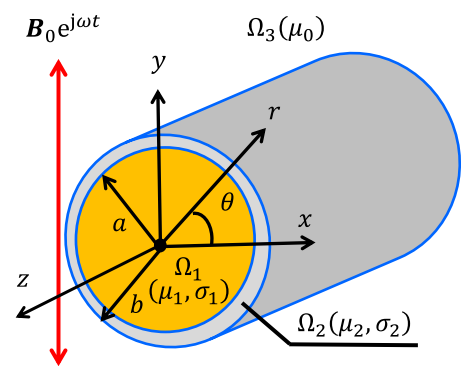

(a) MSW in time-harmonic magnetic induction

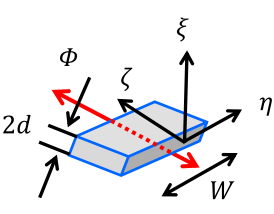

(b) Thin slab model of shielding layer
Fig.1 Magnetically shielded wire (MSW) and local coordinate system introduced in a thin slab which models the shielding layer.

the eddy currents in MSW. Let us consider an infinitely long MSW, shown in Fig.1(a), immersed in a uniform timeharmonic magnetic induction $\boldsymbol{B}_{0} \mathrm{e}^{\mathrm{j} \omega t}$. The fields obey

$$
\begin{gathered}
\frac{\partial^{2} E_{z}}{\partial r^{2}}+\frac{1}{r} \frac{\partial E_{z}}{\partial r}+\frac{1}{r^{2}} \frac{\partial^{2} E_{z}}{\partial \theta^{2}}+k_{i}^{2} E_{z}=0 \\
H_{\theta}=-\frac{\mathrm{j}}{\omega \mu_{i}} \frac{\partial E_{z}}{\partial r}
\end{gathered}
$$

where $\quad k_{3}=0$ in $\Omega_{3}, \quad k_{i}=\sqrt{-\mathrm{j} \omega \sigma_{i} \mu_{i}}=(1-\mathrm{j}) / \delta_{i} \in$ $\mathbb{C}$ in $\Omega_{i}, i=1,2$, and $\delta_{i}$ denotes the skin depth. Imposing the boundary conditions to the solutions to (1), e.g. [6], on the interfaces between $\Omega_{1}$ and $\Omega_{2}, \Omega_{2}$ and $\Omega_{3}$, we obtain a system of equations for the unknown coefficients

$$
\begin{gathered}
{\left[\begin{array}{cccc}
J_{1}\left(k_{1} a\right) & -J_{1}\left(k_{2} a\right) & -N_{1}\left(k_{2} a\right) & 0 \\
J_{1}^{\prime}\left(k_{1} a\right) & -\tau_{12} J_{1}^{\prime}\left(k_{2} a\right) & -\tau_{12} N_{1}^{\prime}\left(k_{2} a\right) & 0 \\
0 & b J_{1}\left(k_{2} b\right) & b N_{1}\left(k_{2} b\right) & -1 \\
0 & b^{2} J_{1}^{\prime}\left(k_{2} b\right) & b^{2} N_{1}^{\prime}\left(k_{2} b\right) & \mu_{2} /\left(k_{2} \mu_{0}\right)
\end{array}\right]\left[\begin{array}{c}
c_{1} \\
c_{2} \\
c_{3} \\
c_{4}
\end{array}\right]} \\
=\left[\begin{array}{c}
0 \\
0 \\
\mathrm{j} \omega b^{2} B_{0} \\
\mathrm{j} \omega b^{2} B_{0} \mu_{2} /\left(k_{2} \mu_{0}\right)
\end{array}\right]
\end{gathered}
$$

where $\tau_{12}=\delta_{1} \mu_{1} / \delta_{2} \mu_{2}$, and $J_{1}, N_{1}$ denote the first order Bessel and Neumann functions, and the prime denotes the derivative with respect to the argument. The coefficient $c_{4}$ is relevant to the magnetic dipole of MSW. Namely, the magnetization of MSW is expressed as $M=-\mathrm{j} 2 c_{4} /\left(\mu_{0} \omega b^{2}\right)$ 
$[4,5]$. On the other hand, from the analogy with a magnetic cylinder immersed in a uniform magnetostatic field, the magnetization of a magnetic-conductive cylinder in timeharmonic field is expressed as

$$
M=2 \frac{B_{0}}{\mu_{0}} \frac{\dot{\mu}_{r}-1}{\dot{\mu}_{r}+1}
$$

where $\dot{\mu}_{r}$ denotes the relative complex permeability. By inserting $M$ obtained from (2) into (3), we have an analytical expression of $\dot{\mu}_{r}$. It is found, however, that the matrix in (2) becomes ill-conditioned for large values of $k_{i}$. To understand the reason, we consider the asymptotic expansion

$$
J_{1}\left(k_{i} a\right) \sim \sqrt{\frac{2}{\pi k_{i} a}} \cos \left(k_{i} a-\frac{3}{4} \pi\right) .
$$

We can see from (4) that $J_{1}\left(k_{i} a\right)$ is proportional to $\mathrm{e}^{a / \delta_{i}}$, and $\mathrm{e}^{a / \delta_{2}}$ can be much larger than $\mathrm{e}^{a / \delta_{1}}$ when $\Omega_{1}, \Omega_{2}$ are filled with, e.g., copper and iron whose ratio of skin depths is typically more than 4 . When $a / \delta_{1}=10$, for example, $\mathrm{e}^{a / \delta_{1}} \approx$ $2 \times 10^{4}$, while $\mathrm{e}^{a / \delta_{2}} \approx 2 \times 10^{17}$. Similar situations can happen in other terms in (2). This gives rise to large errors in $\dot{\mu}_{r}$ even when we analytically solve (2). We will introduce a method to circumvent this difficulty in the next section.

\section{PROPOSED APPROACH}

\section{A. Complex permeability of magnetic-conductive plate}

The thickness $b-a$ of the shielding layer in MSW, shown in Fig.1 (a), is usually much smaller than the wire radius $a$. The curvature of the layer could be, therefore, neglected in good approximation. Under this assumption, the magneticconductive layer in Fig.1 (a) can be approximated as a slab as shown in Fig. 1 (b). This approximation has been used for modeling of thin magnetic shells [7, 8]. The quasi-static electromagnetic field in the slab obeys the one-dimensional Helmholtz equation

$$
\frac{\mathrm{d}^{2} H_{\zeta}}{\mathrm{d} \xi^{2}}+k_{2}^{2} H_{\zeta}=0
$$

where $\xi, \zeta$ are the axes in the radial and tangential directions of the layer. Solving (5) under the boundary conditions $H_{\zeta}( \pm d)=H_{0}, d=(b-a) / 2$, the magnetic flux $\Phi$ can be obtained as

$$
\Phi=W \int_{-d}^{d} \mu_{2} H_{\zeta} \mathrm{d} \xi=\mu_{2} S \frac{\tan \alpha}{\alpha} H_{0}
$$

where $\alpha=k d \in \mathbb{C}, S=2 d W$. The complex permeability of the slab is then given by

$$
\dot{\mu}_{2}(\omega) \equiv \frac{\Phi / S}{H_{0}}=\mu_{2} \frac{\tan \alpha}{\alpha}
$$

which has been used for the eddy current analysis of a steel sheet [7]. Since the eddy current effects are already included in (7), the magnetic-conductive layer can be now treated as an insulator with $\dot{\mu}_{2}$. Note here that $\tan \alpha$ and $\alpha$ in (7) are asymptotically proportional to $\mathrm{e}^{a / \delta_{2}}$. Since these factor cancel out, there is no numerical difficulty in computation of (7).

\section{B. Complex permeability of MSW coil}

As mentioned in Section I, the complex permeability of an MSW coil with an insulating magnetic shield has been given in [5]. On the other hand, by introducing $\dot{\mu}_{2}$, we can treat the magnetic-conductive shield as an insulating one as shown in Section II.A. The formulation in [5] is, therefore, valid for the analysis of an MSW coil when using $\dot{\mu}_{2}$ instead of $\mu_{2}$. For completeness of this paper, we briefly give the formulation for MSW with an insulating magnetic layer below.

We assume that $\sigma_{2}=0$. By imposing the tangential continuity of $E_{z}$ and $H_{\theta}$ on the interfaces, we obtain a system of equations of the form

$$
\left[\begin{array}{cccr}
J_{1}\left(k_{1} a\right) & -1 / a & -a & 0 \\
k_{1} v_{1} J_{1}^{\prime}\left(k_{1} a\right) & 1 / a^{2} & -1 & 0 \\
0 & 1 & b^{2} & -1 \\
0 & v_{2} & -v_{2} b^{2} & -1
\end{array}\right]\left[\begin{array}{l}
c^{\prime} \\
c_{2}{ }^{\prime} \\
c_{3}{ }^{\prime} \\
c_{4}{ }^{\prime}
\end{array}\right]=\left[\begin{array}{c}
0 \\
0 \\
\mathrm{j} \omega b^{2} B_{0} \\
-\mathrm{j} \omega b^{2} B_{0}
\end{array}\right]
$$

where $v_{1}=\dot{\mu}_{2}(\omega) / \mu_{1}, v_{2}=\mu_{0} / \dot{\mu}_{2}(\omega)$. Here the coefficient $c_{4}{ }^{\prime}$, which corresponds to $c_{4}$ in (2), is relevant to the dipole field. By solving (8), we can obtain the magnetization

$$
M=2 \frac{B_{0}}{\mu_{0}} \frac{\lambda_{1} \frac{J_{1}\left(z_{1}\right)}{z_{1} J_{1}^{\prime}\left(z_{1}\right)}+v_{1} \lambda_{2}}{\lambda_{3} \frac{J_{1}\left(z_{1}\right)}{z_{1} J_{1}^{\prime}\left(z_{1}\right)}+v_{1} \lambda_{4}}
$$

where $\quad \lambda_{1}=-h_{1} v_{2}-h_{2}, \lambda_{2}=h_{2} v_{2}+h_{1}, \lambda_{3}=h_{1} v_{2}-$ $h_{2}, \lambda_{4}=-h_{2} v_{2}+h_{1}, h_{1}=a^{2}-b^{2}, h_{2}=a^{2}+b^{2}$ and $z_{1}=$ $k_{1} a \in \mathbb{C}$. Comparing (3) with (9), we obtain $\dot{\mu}_{r}$. Note here that (9) again leads to no numerical difficulty because the factor, $\mathrm{e}^{a / \delta_{1}}$, relevant to $z_{1}$ cancels out.

The macroscopic permeability of multi-turn coils composed of MSW can be obtained from the extended Ollendorff formula $[4,10]$ given by

$$
\left\langle\dot{\mu}_{r}\right\rangle=1+\frac{\eta\left(\dot{\mu}_{r}-1\right)}{1+N(1-\eta)\left(\dot{\mu}_{r}-1\right)}
$$

where $\eta, N$ denote the volume fraction and diamagnetic constant, and the latter is set to $1 / 2$ for round MSW. Using (10), the MSW coil can be modeled as a uniform material. When we consider the magnetic saturation in the magnetic shield, we would evaluate the convolution integral including (10) for conversion from the frequency domain to time domain. The total power $P$ can be evaluated from the energy conservation law

$$
\begin{aligned}
P=\frac{\mathrm{j} \omega}{2} \int_{\Omega} & \mu|\boldsymbol{H}|^{2} \mathrm{~d} v \\
& +\frac{R_{0} z_{1} J_{0}\left(z_{1}\right)}{4 J_{1}\left(z_{1}\right)}|I|^{2}+\mathrm{j} \omega \dot{\mu}_{2 r} \mu_{0} l \pi\left|\frac{I}{2 \pi}\right|^{2} \log \left(\frac{b}{a}\right)
\end{aligned}
$$

where $l$ is the coil length. The first term in (11) includes the eddy current loss due to the proximity effect and time 
variation in the stored magnetic energy in air region $\Omega_{3}$, while the second term represents the eddy current loss due to the skin effect. The magnetic energy stored in a magnetic shield is expressed in the third term. If there are no conductors around MSW, then the magnetostatic equation is solved, else the quasi-static Maxwell equation is solved to consider the eddy currents in the external conductors, where the magnetic permeability is set to $\langle\dot{\mu}(\omega)\rangle$ in the MSW region. The impedance $Z$ of MSW coil is calculated from $Z=2 P /|I|^{2}$.

\section{NUMERICAL RESULTS}

\section{A. Frequency characteristics of complex permeability}

We first consider MSW whose specification is summarized in Table I, where the shield permeability, $\mu_{2 r}$, is assumed rather small for computation of (2). The frequency characteristics of complex permeability obtained from the method mentioned in Section II and proposed approach in Section III are plotted in Fig.2, where the abscissa denotes the wire radius $a$ normalized by the skin depth $\delta_{1}$. It can be seen from Fig. 2 that although there are discrepancies between them for the thick layer as shown in (b), as expected, the results obtained by the proposed approach agree well with those obtained by the method in Section II for the thin layer as shown in (a). It is concluded that the proposed method gives accurate results when thickness is sufficiently smaller than the wire radius.

We next consider MSW whose parameters are given in Table II. Now the shield permeability is set higher to consider actual MSW used in industries [1-3]. The method described in Section II breaks down for this setting. In contrast, the frequency dependence, plotted in Fig.3, can be obtained by the proposed method without numerical difficulties.

The impedance, $Z$, of a 9-turn MSW coil is computed by the proposed method and conventional FEM. The coil region is discretized into rather coarse elements in the former analysis, while it is descritized into very fine elements so that their size is smaller than the skin depth, as shown in Fig.4. For the coil,

TABLE I

SPECIFICATION OF MAGNETIC SHIELDED WIRE WITH LOW PERMEABILITY

\begin{tabular}{c|c||c|c||c|c}
\hline \hline$a[\mathrm{~mm}]$ & $0.50,1.00$ & $\mu_{1 r}$ & 1 & $\sigma_{1}[\mathrm{~S} / \mathrm{m}]$ & $5.76 \times 10^{7}$ \\
$b[\mathrm{~mm}]$ & 1.10 & $\mu_{2 r}$ & 10 & $\sigma_{2}[\mathrm{~S} / \mathrm{m}]$ & $1.0 \times 10^{4}$ \\
\hline \hline
\end{tabular}

SPECIFICATION OF MAGNETIC SHIELDED WIRE

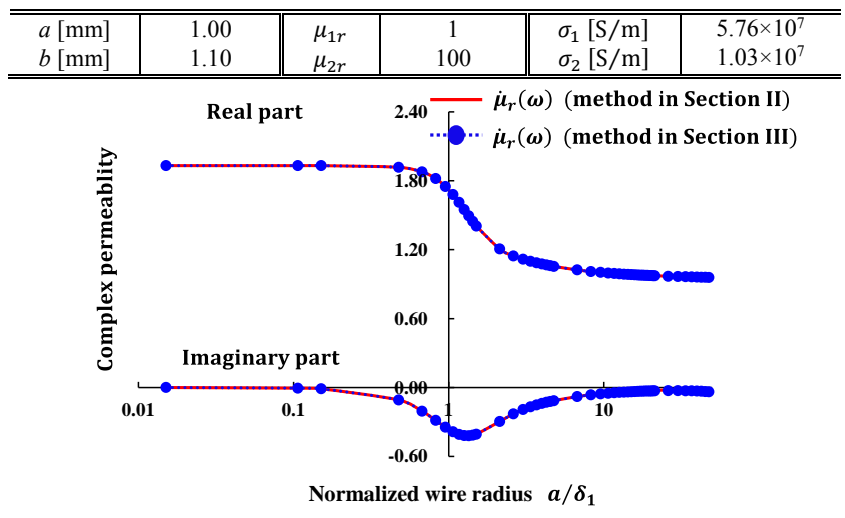

(a) $a=1.00 \mathrm{~mm}$

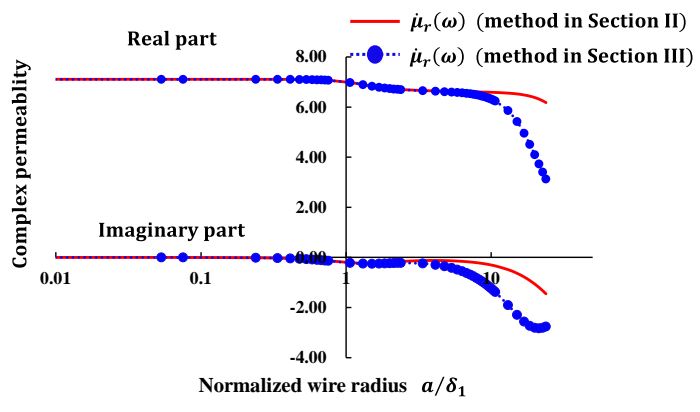

(b) $a=0.50 \mathrm{~mm}$

Fig.2 Profiles of complex permeability for setting in Table I

\section{B. Impedance of MSW coil}

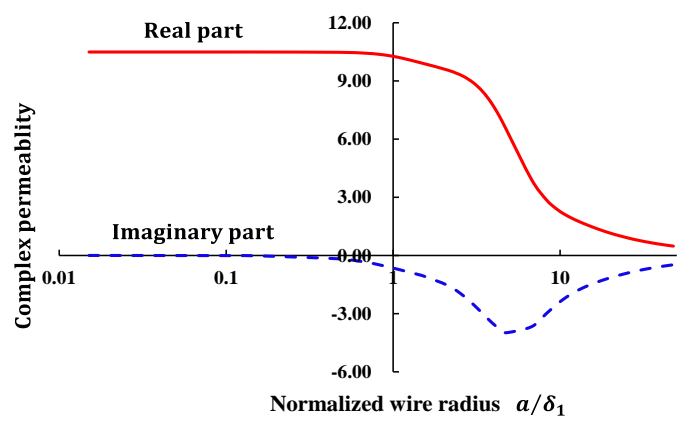

Fig.3 Profiles of complex permeability for setting in Table II

we assume MSW whose specification is in Table I, where $a=$ $1 \mathrm{~mm}$, so that the conventional FE analysis is executable. There are 360 and 661,358 elements in the coil region for the former and latter analyses, respectively. In Fig.5, $Z$ is plotted against the normalized wire radius $a / \delta_{1}$ for driving frequency ranging from $1 \mathrm{~Hz}$ to $1 \mathrm{MHz}$. It is found that the maximum discrepancy is less than $2.2 \%$ and $4.0 \%$ in the real and imaginary parts, respectively.

\section{OPTIMAL STRUCTURE OF MSW}

On the basis of the proposed method, we seek for the optimal MSW structure. The wire radius $b$ and driving frequency are fixed to $1.1 \mathrm{~mm}, 200 \mathrm{kHz}$, respectively. When the radius of the inner conductor, $a$, increases, the DC resistance of the whole wire decreases, while the eddy current loss increases because the shield thickness decreases. For this reason, there would exist an optimal radius for $a$.

The dependences of the AC resistance on $a / b$ for different shielding conductivities are plotted in Fig.6. The ratio $a / b$ which gives the mimimum $\mathrm{AC}$ resistance is found to increase with $\sigma_{2} ; a / b \approx 0.864,0.981,0.991$ for $\sigma_{2}=$ $0,10^{6}, 10^{7} \mathrm{~S} / \mathrm{m}$.

\section{EFFECTIVENESS OF MSW}

Finally, we compare the impedance $Z$ of MSW with that of a usual wire without shields. We consider a 25 -turn MSW coil whose spesification is summarized in Table III, where $\mu_{2}=$ $1.0, \sigma_{2}=0$ for the latter wire. The real part of $Z$ is plotted against frequency in Fig.7. It is found that the reduction of the $\mathrm{AC}$ resistance, $\operatorname{Re}(Z)$, by $\mathrm{MSW}$ becomes significant as frequency increases. In particular, the loss of MSW is almost the half in comparison with that of the usual wire at $1 \mathrm{MHz}$. 


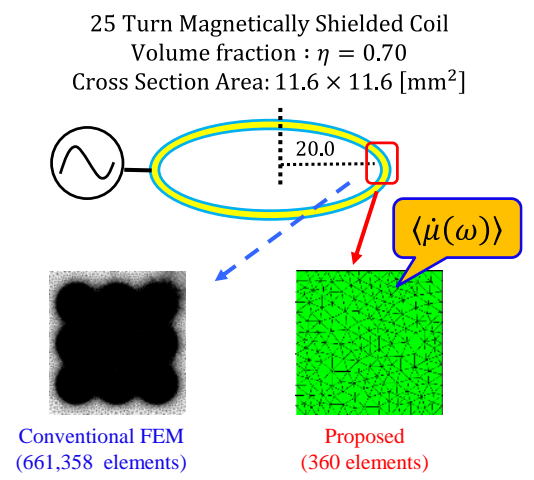

Fig.4 Magnetically shielded coil. In proposed method, the coil region is discretized by coarse elements with the macroscopic complex permeability.

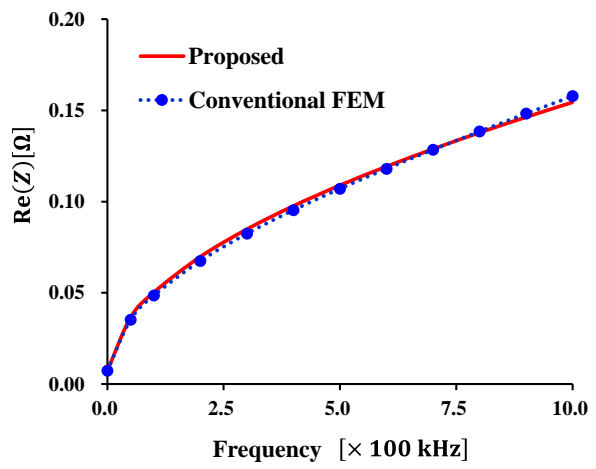

(a) real part

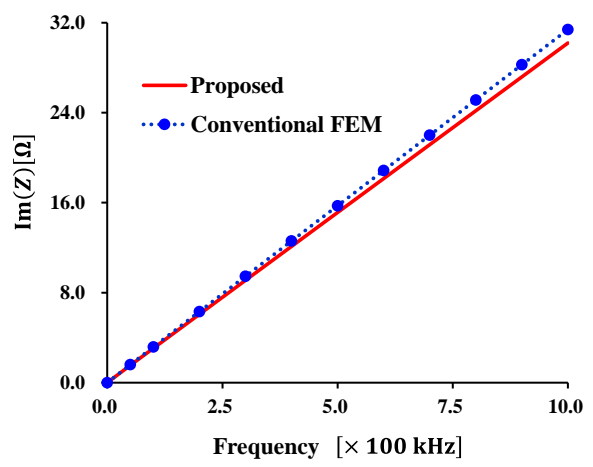

(b) imaginary part

Fig.5 Frequency characteristic of coil impedance. In these figure, the abscissa is normalized by $100 \mathrm{kHz}$.

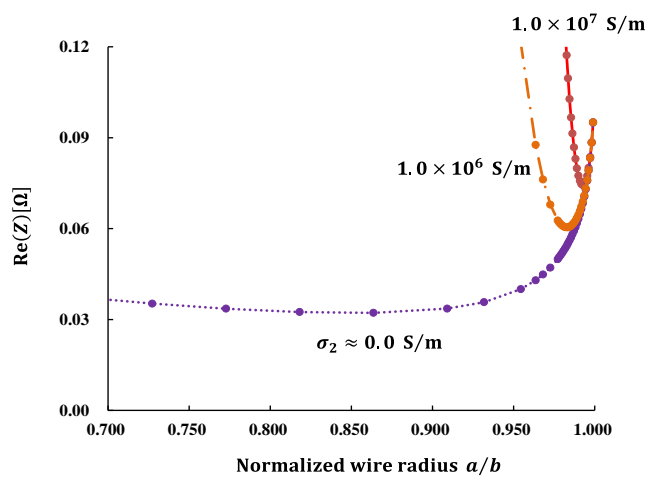

Fig.6 Dependence of the AC resistance of MSW on normalized wire radius $a / b$ for different shield conductivity $\sigma_{2}$.

\section{CONCLUSIONS}

A homogenization-based FEM for the analysis of MSW has been proposed. To avoid the numerical difficulty, the complex permeability of a magnetic-conductive slab is used for that of the shielding layer in MSW assuming that its thickness is sufficiently smaller than the wire radius. An MSW coil can be modeled as a uniform material with macroscopic permeability which is obtained by inserting the complex permeability into the Ollendorff formula. From the numerical results, the proposed method has been shown to be accurate. The optimal structure of MSW has been obtained using the proposed method. Reduction of eddy current loss by MSW becomes significant as frequency increases. Comparison of the numerical results with measurement remains for our future work.

TABLE III

SPECIFICATION OF MAGNETIC SHIELDED WIRE

\begin{tabular}{c|c||c|c||c|c}
\hline \hline$a[\mathrm{~mm}]$ & 0.144 & $\mu_{1 r}$ & 1 & $\sigma_{1}[\mathrm{~S} / \mathrm{m}]$ & $5.76 \times 10^{7}$ \\
$b[\mathrm{~mm}]$ & 0.15 & $\mu_{2 r}$ & 100 & $\sigma_{2}[\mathrm{~S} / \mathrm{m}]$ & $1.03 \times 10^{7}$ \\
\hline \hline
\end{tabular}

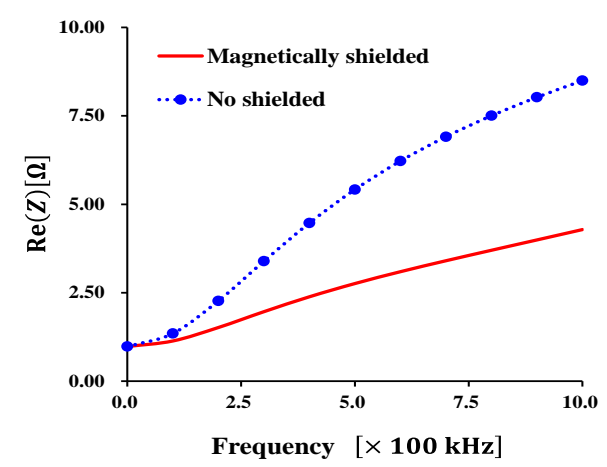

Fig.7 Comparison of frequency characteristic of impedance.

\section{REFERENCES}

[1] T. Mizuno, et. al.,. "Reduction of proximity effect in coil using magnetoplated wire", IEEE Trans. Magn., vol. 43, no. 6, 2003, pp. 539548.

[2] A. Podoltsev and B. Lebedev, "Analysis of effective resistance and eddy current losses in multiturn winding of high-frequency magnetic components," IEEE Trans. Magn., vol. 39, no. 1, , 2003, pp. 539-548.

[3] J. Gyselinck and P. Dular, "Frequency-domain homogenization of bundles of wires in 2-D magnetodynamic FE calculations," IEEE Trans. Magn., vol. 41, no. 5, 2005 pp. 1416-1419.

[4] H. Igarashi, "Semi-Analytical Approach for Finite Element Analysis of Multi-turn Coil Considering Skin and Proximity Effects", IEEE Trans., Magn. vol.53, no. 1, 7400107, 2017.

[5] S. Fujita, H. Igarashi, "Finite Element Analysis of Multi-turn Magnetoplated Coils Using Homogenization Method", IEEJ, Trans. on Power and Energy, vol.137, No.3, 2017, pp.1-7 (in Japanese).

[6] M. Abramowitz and I.A. Stegun (eds.), Handbook of mathematical functions, Dover, 1965.

[7] L. Krähenbühl, D. Muller, "Thin layers in electrical engineering.Examples of shell models in analyzing eddy-currents by boundary and finite element methods," IEEE Trans. Magn., vol. 29, no. 2, 1993, pp. 1450-1455.

[8] I. D. Mayergoyz and G. Bedrosian, "On calculation of 3-D eddy currents in conducting and magnetic shells," IEEE Trans. Magn., vol. 31, no. 3, 1995 pp. 1319-1324.

[9] T. Miyazaki, et.al, "Equivalent circuit modeling of dynamic hysteric property of silicon steel under pulse width modulation excitation", $J$. Appl. Phys., 117, 2015, 17D110.

[10] F. Ollendorff, "Magnetostatik der Massekerne," Arch. f. Eledtrotechnik., 25, 1931, pp. 436-447. 